\title{
Setor de agências de internet no Brasil: análise de concorrência a partir de simulação dinâmica de sistemas
}

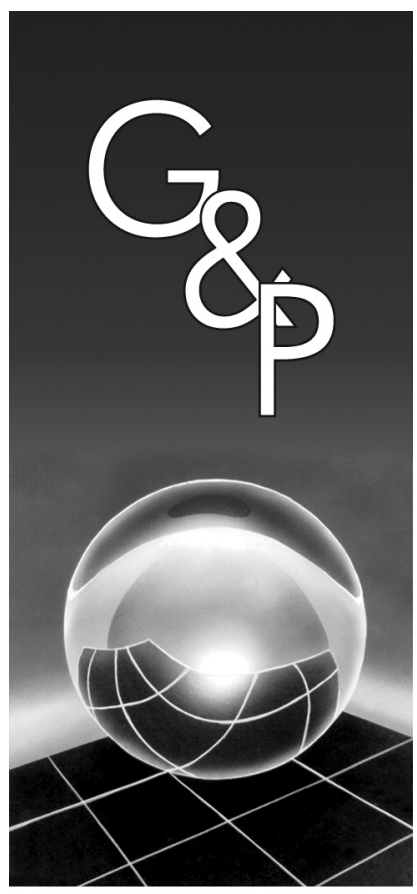

Antony José Souza Martins

Paulo Furquim de Azevedo

\section{Resumo}

Este artigo tem por objetivo analisar a participação de mercado dos diferentes grupos estratégicos que compõem o setor de agências de internet no Brasil, bem como as mudanças decorrentes de alterações nas variáveis críticas de concorrência, tais como preço, investimento em propaganda, investimento em tecnologia e disponibilidade de mão-de-obra. Para tanto, será aplicado o método de simulação computacional, utilizando-se dinâmica de sistemas. A adoção desta técnica permitiu identificar padrões contra-intuitivos, o que se revelou acertado, principalmente no que concerne às análises sobre mudanças no grau de diferenciação entre os diferentes grupos estratégicos analisados.

Palavras-chave: Internet. Indústria de software. Dinâmica de sistemas. Bertrand diferenciado.

\section{Introdução}

Este artigo tem por objetivo analisar a participação de mercado dos diferentes grupos estratégicos que compõem o setor de agências de internet no Brasil, bem como as mudanças decorrentes de alterações nas variáveis críticas de concorrência, tais como: preço, investimento em propaganda, investimento em tecnologia e disponibilidade de mão-de-obra. Para tanto, será aplicado o método de simulação computacional, utilizando-se dinâmica de sistemas.

Em aproximadamente dez anos, houve um intenso desenvolvimento do setor de agências de internet no Brasil, com o amadurecimento deste novo setor econômico e a ampliação dos dispositivos interativos empregados. A atividade prestada por esse setor ganhou papel fundamental para as estratégias de todos os demais setores econômicos.

Nesse estudo, foi empregada uma abordagem de simulação computacional, em particular dinâmica de sistemas, por dois motivos. Por um lado, existem poucas informações disponíveis e confiáveis sobre o setor, pois trata-se de um ramo novo e ainda em processo de consolidação. Essa escassez de dados e a ausência de informações secundárias impossibilitam a adoção de análises quan- titativas por meio de técnicas econométricas. Por outro lado, a simulação matemática por um modelo estruturalmente fechado não seria adequada para tratar situações complexas como, nesse caso, a análise do comportamento de diferentes grupos estratégicos de uma mesma indústria. A simulação por dinâmica de sistemas permite maior flexibilidade para acomodar diferentes cenários e estruturas de indústria, sendo, portanto, mais adequada ao propósito deste artigo. A adoção desta técnica permitiu identificar padrões contra-intuitivos, principalmente no que concerne às análises sobre mudanças no grau de diferenciação entre os diferentes grupos estratégicos analisados.

O trabalho divide-se em quatro seções, incluindo esta introdução. A segunda seção apresenta as características gerais do setor de agências de internet, a fim de identificar os principais elementos para o desenho do modelo de simulação. Na terceira seção, são detalhados os modelos desenvolvidos e o resultado das principais simulações. Por fim, a quarta seção sumaria os principais resultados, bem como traz recomendações para extensões deste estudo. 


\section{Agências de internet: características gerais}

\subsection{Surgimento do setor de agências de internet}

A internet é um conjunto de redes de computadores interligadas que têm em comum um conjunto de serviços. Os usuários conectados a esta rede podem usufruir dos serviços de informação e comunicação de alcance mundial (LAUDON; LAUDON, 1999).

A internet surgiu na época da Guerra Fria com o nome de Arpanet e com a finalidade de manter ativa a comunicação das bases militares dos Estados Unidos, caso os servidores do Pentágono fossem atingidos por um ataque nuclear. Com o fim da Guerra Fria, os cientistas passaram a utilizar a internet para troca de informações, e mais tarde trouxeram para a rede as universidades americanas, as quais, sucessivamente, incluíram universidades de outros países, permitindo que pesquisadores do mundo todo pudessem se comunicar.

Em 1992, com o surgimento da World Wide Web, o que antes era apenas uma rede de comunicação, tornou-se uma mídia, uma plataforma de relacionamento e um canal de vendas. Essa mudança foi possível pois esse novo protocolo de comunicação permitiu a incorporação de imagens e sons, tornando a rede mais atraente. Desde então, a internet experimentou um crescimento exponencial do número de redes, servidores, usuários e volume de tráfego.

A história da internet no Brasil começou em 1991 com a Rede Nacional de Pesquisa (RNP), uma operação acadêmica subordinada ao Ministério de Ciência e Tecnologia. Em meados de 1994, a Embratel lançou o serviço experimental de acesso a internet, e em 1995 foi realizada a abertura da exploração comercial da internet para o setor privado (CGI, 2005).

Nesse momento, as corporações perceberam na internet novas oportunidades de negócios e procuraram se inserir no mundo virtual. Surgiram, então, as agências de internet, aptas a realizar essa inserção, explorando as oportunidades da rede.

\subsection{Condições básicas da oferta}

O uso intensivo de tecnologia é uma das características determinantes do setor de agências de internet, razão pela qual é imprescindível analisar seu impacto na oferta dos serviços prestados. Os investimentos em tecnologia são constantes, pois o setor se expande a altas taxas, e ainda porque há uma grande velocidade de mudança nos padrões tecnológicos.

Essa evolução rápida e constante nos padrões tecnológicos pode ser explicada pela intensa rivalidade entre os fornecedores de hardwares e softwares para internet, tais como Sun, Microsoft, Oracle, Adobe e Macromedia, que vivem aquilo que Shapiro e Varian (1999, p. 299) denominam 'guerra de padrões', ou seja, duas ou mais empresas com tecnologias novas e incompatíveis lutando para se tornarem um padrão de mercado. Essa guerra, por um lado, obriga tais empresas a investirem maciçamente em pesquisa e desenvolvimento de novos produtos e, por outro, torna esses novos produtos disponíveis e acessíveis para o mercado. Esta característica, conforme detalhado a seguir, torna a disponibilidade de recursos humanos qualificados o principal recurso de concorrência, visto que a estrutura tecnológica é continuamente transformada pela 'guerra de padrões'.

O capital na forma de estoques também não é relevante em comparação com o domínio dos ativos intangíveis e recursos humanos, o que decorre do fato de a produção das agências de internet ser normalmente desenvolvida após a venda. Desse modo, o setor apresenta alta eficiência na alocação dos recursos disponíveis e necessários à consecução de sua atividade, não estando sujeito a problemas de flutuação de estoques.

Essa eficiência é, entretanto, limitada pela capacidade máxima de produção de cada agência, esta determinada pelo volume de projetos que a equipe de gestores é capaz de atender simultaneamente sem perder qualidade. Esta equipe, por sua vez, não pode ser rapidamente aumentada em razão dos conhecimentos específicos e da experiência exigida desses profissionais. Quando esse limite é superado perde-se eficiência na alocação dos recursos e qualidade na prestação do serviço.

Por se tratar de serviços especializados, que podem representar elevados investimentos por parte dos compradores, os principais ativos de uma agência de internet são associados à credibilidade na empresa. Como conseqüência, tais ativos são, em geral, intangíveis, associados à marca, à carteira de clientes, ao histórico de sucesso, à metodologia e processo de desenvolvimento de trabalho e, ainda, à capacidade de inovação criativa e tecnológica (LOVELOCK, 1996).

O progresso técnico é um elemento fundamental para a indústria em estudo, dado o intensivo uso de tecnologia e as novas possibilidades que dela decorrem para o setor, com profundos impactos sobre ele. Originalmente, a comunicação interativa possuía como principal meio a internet. Com novas tecnologias disponíveis, esses meios foram muito ampliados, destacando-se, por exemplo, handhelds, celulares e TV interativa. A perspectiva é de contínua ampliação, viabilizando-se, no futuro, a comunicação interativa por inúmeros dispositivos, tais como geladeiras, automóveis e microondas.

O setor de agências de internet caracteriza-se pelo emprego intensivo de capital humano. Por se tratar de uma indústria de informações e de serviços, pois sua atividade baseia-se na prestação de serviços lastreados em conhe- 
cimento e informação, o setor emprega especialmente o que Laudon e Laudon (1999, p. 308) denominam de "trabalhador do conhecimento".

Os "trabalhadores do conhecimento" se distinguem dos outros tipos de trabalhadores pelo alto nível de instrução exigido no desempenho de suas tarefas e pelo alto componente criativo de seu trabalho. Esses trabalhadores caracterizam-se por possuírem grande base de conhecimento, que os habilita a atuarem de maneira autônoma e criativa, com capacidade de adaptação aos mais diversos tipos de cenários e, ainda, por possuírem expressivos potenciais de aperfeiçoamento em função das experiências práticas vivenciadas (LAUDON; LAUDON, 1999, p. 309).

As agências de internet empregam basicamente dois tipos de profissionais com as características acima descritas: profissionais de tecnologia (engenheiros, cientistas de computação e analistas de sistemas) e de criação (publicitários, jornalistas, desenhistas industriais e ilustradores). Esses profissionais possuem profundos conhecimentos específicos, e diferentes entre si, necessários à prestação dos serviços.

Uma parcela das agências tem seu foco em projetos de tecnologia, optando, portanto, por profissionais dessa área. De outro lado, existem agências voltadas para a criação, e seu elenco de profissionais é adequado para esse enfoque. Contudo, um terceiro grupo de agências procura desenvolver projetos híbridos que envolvam os dois recursos. Esse grupo necessita de um terceiro tipo de profissional, o gestor de projeto, cuja competência principal é planejar e definir estratégias para agrupar os conhecimentos antes fragmentados, de tecnologia e criação, de forma a maximizar a eficiência na alocação dos recursos.

Além dos conhecimentos técnicos já referidos, os profissionais com experiência na área adquirem, ao longo do tempo, conhecimentos muito específicos, mas genéricos entre os concorrentes do setor. Assim, esses trabalhadores são valorizados e disputados no mercado, tornando os custos para seu recrutamento e retenção bastante elevados. Exige-se, assim, das agências que compõem o setor, a prática de políticas de recursos humanos diferenciadas e atraentes (salários atrativos, benefícios, processos de trabalho flexíveis e dinâmicos, entre outros) para a manutenção de seus profissionais (MILGROM; ROBERTS, 1992, p. 344).

Portanto, ao contrário do que ocorre com o capital, a organização do trabalho neste setor é um dos fatores principais para determinar a estrutura de custos e, conseqüentemente, os preços praticados.

\subsection{Condições básicas da demanda}

As condições básicas da demanda dependem fundamentalmente do processo de compra, da pequena disponibilidade de substitutos próximos e das externalidades de rede, pontos estes que são detalhados a seguir.

Ao comprar soluções de comunicação interativa, as empresas têm como objetivos: trabalhar a imagem da marca, criar canais de relacionamento pela internet, vender produtos ou compartilhar informações. Elas podem buscar soluções de criação ou de tecnologia. No primeiro caso, o foco é a consultoria em comunicação interativa para o desenvolvimento de soluções criativas (websites, portais corporativos, hotsites, intranets, extranets, e-mail marketing, entre outros). No segundo, o foco é o desenvolvimento de aplicações para suportar as soluções de comunicação (gerenciadores de conteúdo, gerenciadores de e-mail marketing, lojas virtuais, tracking de usuários, entre outros).

O processo de compra representa um custo alto para o comprador, além de ser bastante longo, podendo durar entre três e seis meses. O elevado custo se explica porque a empresa compradora envolve, em geral, diversos departamentos, sendo usual a formação de comitês de decisão. Por esse motivo, as empresas não costumam fazer mais de uma concorrência por ano e, em regra, a compra dos serviços de agências de internet é pouco freqüiente.

Dentre os departamentos que usualmente se envolvem na aquisição dos serviços, destacam-se: os de marketing, comunicação, recursos humanos, tecnologia e compras. Normalmente, a concorrência se inicia com as áreas de marketing e comunicação. Os profissionais do departamento de TI, por sua vez, definem pré-requisitos e processos necessários para a implementação dos projetos. O departamento de compras funciona como influenciador ou decisor. Nos casos em que existe um comitê, geralmente ele é composto por representantes de diversas áreas da organização (marketing, comunicação, finanças, recursos humanos, tecnologia e compras), além de ser comum o envolvimento de altos executivos da empresa.

Os principais substitutos do serviço de comunicação interativa são oferecidos pelos setores de comunicação tradicional (comunicação off-line), destacando-se a propaganda (TV, rádio, revista e jornal), ações de marketing direto (malas diretas impressas, revistas e brindes) e promoções nos pontos de venda.

Observa-se, porém, que esses substitutos não possuem os mesmos recursos oferecidos pelo setor de agências de internet, destacadamente a interatividade e o alcance espacial e temporal desta mídia. Portanto, os resultados efetivamente produzidos por um e outro não são similares. Acrescente-se que os substitutos não oferecem uma relação preço/desempenho favorável e as perspectivas futuras não indicam mudança nesse quadro. Por essas razões, a substitutibilidade é baixa e os substitutos não exercem um alto poder de concorrência (PORTER, 1986, p. 40). Como conseqüência, a elasticidade-preço 
da demanda tende a ser baixa, possibilitando maiores margens, a depender do grau de concorrência.

Constata-se, no Brasil, altas taxas de crescimento do número de usuários de internet, de computadores e de empresas conectadas (CARPANEZ, 2005). A difusão de computadores pessoais e de acesso à internet veio ressaltar, para o mercado comprador, a importância de investimentos em soluções de comunicação interativa. Esse é o substrato que permite ao setor de agências de internet também apresentar altas taxas de crescimento.

É importante salientar que o consumo de serviços baseados na internet é afetado pelo que se denomina usualmente "externalidade de rede", em que o consumo de uma pessoa influencia diretamente a utilidade de outra pessoa (VARIAN, 2003, p. 677). Em função desse fenômeno, e do fato acima apontado de que o Brasil já possui um volume considerável de usuários, a perspectiva é de contínuo crescimento do setor a médio prazo.

\subsection{Estrutura de mercado}

O mercado de agências de internet ainda é notadamente marcado por grande fragmentação e pulverização. Contudo, é possível delinear três diferentes grupos dessa indústria, diferenciados pelo foco em criação, tecnologia ou pela conjugação de ambas as competências. Em cada um desses grupos existe um grande número de agências com características semelhantes concorrendo entre si. Os preços de um grupo, por sua vez, são definidos em razão dos preços praticados pelos grupos concorrentes.

As características acima descritas, quais sejam, pequeno número de grupos estratégicos, serviços diferenciados entre os grupos, influências recíprocas sobre os preços praticados por cada grupo, são semelhantes às do modelo de Bertrand diferenciado, tornando adequada sua utilização para a modelagem desse trabalho. Em estruturas de mercado baseadas neste modelo, as empresas adotam os preços, e não as quantidades produzidas, como as variáveis de escolha (SHY, 1995, p. 139). De fato, no setor de agências de internet, a utilização do preço como variável de escolha decorre da incapacidade das firmas alterarem sua estrutura de produção no curto prazo, bem como do baixo custo e da rapidez para mudança de preços.

Os três grupos constituem, na terminologia de Porter (1986, p. 133), grupos estratégicos, uma vez que constituem um "grupo de empresas em uma indústria que está seguindo uma estratégia idêntica ou semelhante ao longo das dimensões estratégicas". O domínio dos ativos relacionados às competências tecnológicas e criativas é o principal elemento para a distinção entre os três grupos, como a seguir explicitado (Figura 1):

a) Grupo 1: Os criativos - empresas com alta capacidade criativa e baixo domínio das tecnologias. Neste grupo, encontram-se os departamentos interativos de agências de publicidade como DM9, AlmapBBDO, Thompson, Ogilvy, entre outras;

b) Grupo 2: Os tecnólogos - empresas com baixa capacidade criativa e alto domínio tecnológico. Neste grupo, encontram-se os integradores de sistemas, como EverSystems, Scopus, Gedas, DBA, entre outras; e

c) Grupo 3: Os híbridos - empresas com alta capacidade criativa e elevado domínio tecnológico. Neste grupo, encontram-se as puras agências de internet, como AgenciaClick, TV1, Tesla, Euro 4D, entre outras.

A principal barreira de mobilidade entre os grupos é a necessidade de capacitação em uma das competências exigidas (criação e tecnologia). É importante destacar, que o simples treinamento da equipe não é suficiente para a migração de um grupo para outro, pois a mudança de cultura e dos processos internos, causada pela inclusão de uma nova competência, impõe obstáculos que, para serem superados, exigem modificações estruturais nos ativos intangíveis de cada grupo.

Em regra, as estratégias de preço de cada um dos três grupos analisados é reflexo do emprego das competências específicas que possuem. Assim, o grupo 1 (os criativos) possui preços vantajosos para projetos que requeiram grande capacidade criativa e pouca aplicação tecnológica. Inversamente, o grupo 2 (os tecnólogos) pode oferecer melhores preços para projetos que empreguem maciçamente tecnologia e requeiram pouca criatividade.

O grupo 3 (os híbridos) é o único grupo apto a desenvolver projetos que envolvam concomitantemente competências criativas e tecnológicas elevadas, razão pela qual o preço não é um fator relevante para a compra. Esses projetos possuem o melhor nível de rentabilidade em todo o setor. Por outro lado, para projetos que envolvam apenas uma das competências, criatividade ou tecnologia, o grupo 3 apresenta os maiores preços, pois

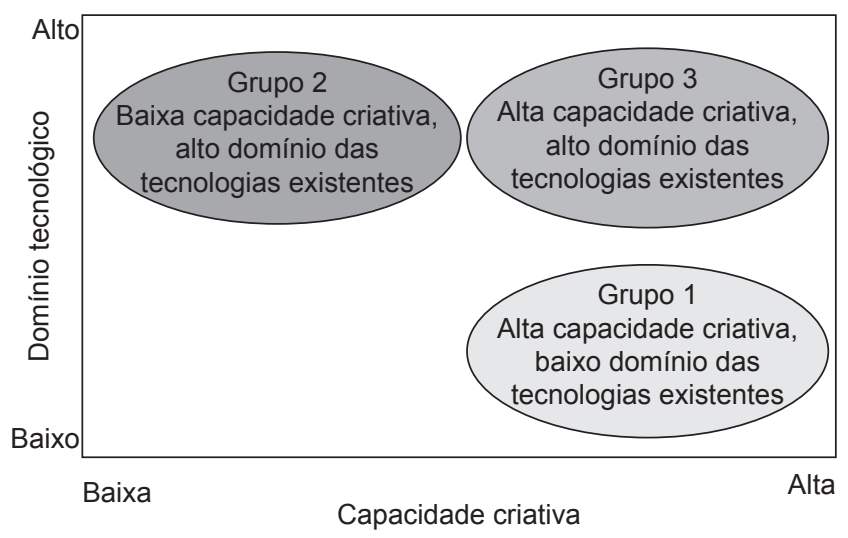

Figura 1. Mapa dos grupos estratégicos. Fonte: Elaborado pelo autor com base na terminologia de Porter (1986). 
sua estrutura de custos, que abrange as duas competências, é mais elevada.

Por fim, é importante destacar que não há concorrência representativa entre os grupos 1 e 2 , pois suas competências são tão distintas que o mercado comprador não percebe estes grupos como concorrentes. Atente-se para o fato de que os comportamentos aqui descritos serão incorporados aos modelos e, por meio de simulações, será possível perceber como variações de preços de cada um dos grupos refletem na sua participação de mercado.

A principal diferença na estratégia de serviço de cada um dos grupos consiste na formação da sua equipe de profissionais. Assim, o grupo 1 investe na formação de uma equipe adequada para o desenvolvimento de soluções criativas, contratando e capacitando profissionais como publicitários, animadores, jornalistas e designers. O grupo 2, por sua vez, concentra os seus esforços na estruturação de uma equipe de perfil tecnológico, recrutando e investindo em engenheiros, analistas e físicos. Finalmente, o grupo 3 requer equipes híbridas, com profissionais das duas áreas e, ainda, um terceiro tipo, já citado, denominado gestor de projetos, que pode ter diversas formações.

As simulações realizadas ao longo desse trabalho demonstram que choques de oferta de mão-de-obra afetam de maneira distinta cada um dos três grupos de empresas, produzindo importantes alterações em suas participações de mercado.

\section{Modelagem por meio de simulação de dinâmica de sistemas}

\subsection{Metodologia}

A metodologia de Dinâmica de Sistemas foi criada nos anos 50 pelo professor Jay W. Forrester, atualmente professor no MIT. Em síntese, essa metodologia usa a simulação computacional para relacionar a estrutura de um sistema com o seu comportamento ao longo do tempo, sendo adequada para casos que envolvam fenômenos complexos, com comportamentos contra-intuitivos, nos quais a variável tempo seja importante.

Nesse estudo, será empregada a abordagem de simulação computacional por dois motivos. Primeiro porque existem poucas informações disponíveis e confiáveis sobre o setor, pois trata-se de um ramo novo e ainda em processo de consolidação. Essa escassez de dados e ausência de informações secundárias impossibilitam a adoção de análises quantitativas através de técnicas estatísticas. Segundo, que uma análise puramente matemática não seria suficientemente poderosa para gerar soluções de situações complexas, como nesse caso, a análise do comportamento de diferentes grupos estratégicos de uma mesma indústria (FORRESTER, 1999, p. 17).

Dentre as técnicas de simulação computacional existentes, adotou-se a dinâmica de sistemas, pois se trata de uma metodologia indicada quando se pretende analisar o comportamento de variáveis ao longo do tempo. Além disso, possui como vantagem a flexibilidade para a construção e a alteração do modelo e seus componentes. Por fim, possibilita uma análise intuitiva do comportamento das variáveis do modelo e de suas inter-relações. Este trabalho utiliza o pacote Vensim $5.0^{\circledR}$, totalmente baseado em dinâmica de sistemas.

Esta seção avalia dinamicamente o comportamento das variáveis críticas do setor de agências de internet no Brasil. Para tanto, foi desenvolvido um modelo a partir das informações levantadas por meio de entrevistas não estruturadas com representantes da indústria e com demandantes, assim como por observação direta do pesquisador. Importante destacar, que foram selecionadas para apresentação apenas as simulações cujos resultados se mostraram mais relevantes e não triviais.

O modelo foi criado com o intuito de analisar o comportamento e as variações nas participações de mercado de cada um dos grupos estratégicos, em função de mudanças na capacidade produtiva dos grupos, do aumento de investimentos em propaganda e/ou em tecnologia, de alterações no mercado de trabalho e de choques externos na economia. Não se fixou como objetivo, portanto, a análise de variáveis financeiras como receitas, custos ou rentabilidades.

Como ponto de partida, foram utilizados os grupos estratégicos anteriormente descritos. Com o intuito de analisar a participação relativa de cada grupo estratégico e, ao mesmo tempo, simplificar a modelagem, cada grupo foi analisado como uma única entidade, de tal modo que deliberadamente não se analisam as relações entre as empresas de cada grupo. Para tal simplificação, o modelo define exogenamente as respostas em termos de preço, contratação de mão-de-obra e estratégias de diferenciação. Uma análise que considera a interação estratégica entre as empresas de cada grupo poderia tornar endógenas variáveis como a resposta a preços e as estratégias de diferenciação. Entretanto, tal procedimento tornaria o modelo excessivamente complexo, o que dificultaria a análise da dinâmica das participações de mercado de cada grupo estratégico, a qual constitui a questão central da pesquisa.

Com o intuito de avaliar o comportamento das variáveis ao longo do tempo, todos os estoques possuem valor inicial igual a zero, no instante $\mathrm{t}=0$, e desta forma, é possível observar a variação da participação de mercado dos diferentes grupos até que seja atingido o equilíbrio. 


\subsection{Modelo de simulação}

\subsubsection{Descrição do modelo}

O modelo descreve um mercado composto por três grupos estratégicos, uma demanda que segue o modelo de concorrência de Bertrand diferenciado, cuja disponibilidade de mão-de-obra é a principal variável para fixação dos preços. Em sua versão mais simples, as variáveis são determinísticas, mas foram também simuladas na incorporação de algumas variáveis estocásticas, a fim de investigar a sensibilidade do modelo a esta característica da modelagem.

O modelo é formado por cinco estoques e cinco fluxos. Os estoques grupo 1, grupo 2 e grupo 3 representam o número de clientes atendidos por cada grupo estratégico e os fluxos 1, 2 e 3 definem, respectivamente, a variação do número de clientes de cada grupo. Os estoques MO-criação e MO-tecnologia denotam a quantidade de mão-de-obra das áreas de criação e tecnologia disponíveis em cada instante do tempo, enquanto os fluxos saída de MO-criação e saída de MO-tecnologia representam as variações dos mesmos estoques.

Além dos estoques e dos fluxos acima descritos, o modelo possui quinze variáveis auxiliares. A auxiliar 'demanda total' se refere à demanda de mercado, enquanto as auxiliares 'demanda 1', 'demanda 2' e 'demanda 3' refletem a demanda particular de cada grupo. Ainda, P1, P2 e P3 definem os preços de cada grupo, a partir, respectivamente, das auxiliares MarkUp 1, MarkUp 2 e MarkUp 3, que representam a margem de lucro. Por sua vez, mkt share 1, mkt share 2 e mkt share 3 denotam a participação de mercado de cada grupo e, finalmente, MO-C-inicial e MO-T-inicial definem o valor da oferta inicial de mão-de-obra de profissionais de criação e tecnologia. A relação entre as variáveis é representada pela Figura 2.

\subsubsection{Mão-de-obra}

Como a competência necessária para a prestação dos serviços necessita de "trabalhadores do conhecimento" (LAUDON; LAUDON, 1999, p. 308) e este tipo de insumo é irreproduzível no curto prazo, pode-se assumir que a oferta inicial de mão-de-obra é função de uma variável exógena e fixa para o período analisado.

A disponibilidade inicial de mão-de-obra de profissionais de criação e de tecnologia é função da variável "Demanda Total", ou seja, a variação desta acarretará mudança proporcional naquela. Isso ocorre porque os profissionais aptos a trabalharem nesse setor possuem a percepção dessa variação de demanda e realizam movimentos migratórios em momentos de crescimento e de retração.

Por observação empírica, constata-se que a oferta de mão-de-obra de profissionais de tecnologia é aproximadamente o dobro da de profissionais de criação, tornando possível definir as seguintes funções:

$$
\begin{aligned}
& \text { MO-T-Inicial }=\text { Demanda Total }{ }^{*} 0.67 \\
& \text { MO-C-Inicial }=\text { Demanda Total }{ }^{*} 0.33
\end{aligned}
$$

Dessafunção,depreende-sequeadisponibilidadeinicial de profissionais de tecnologia será aproximadamente $2 / 3$ da "Demanda Total", enquanto a disponibilidade inicial de profissionais de criação será de $1 / 3$. Assim, garante-se $100 \%$ de atendimento da demanda total.

Inicialmente (em $t=0$ ), os estoques de mão-de-obra de criação e de tecnologia são iguais à sua disponibilidade inicial. A variação desses estoques é inversamente proporcional à variação dos estoques Grupo 1, Grupo 2 e Grupo 3, representativos do número de clientes atendidos por cada grupo, na razão 1:1. Essa situação justifica-se, pois ao contratar profissionais para atender novos clientes, as firmas do setor reduzem a disponibilidade de mão-deobra no mercado.

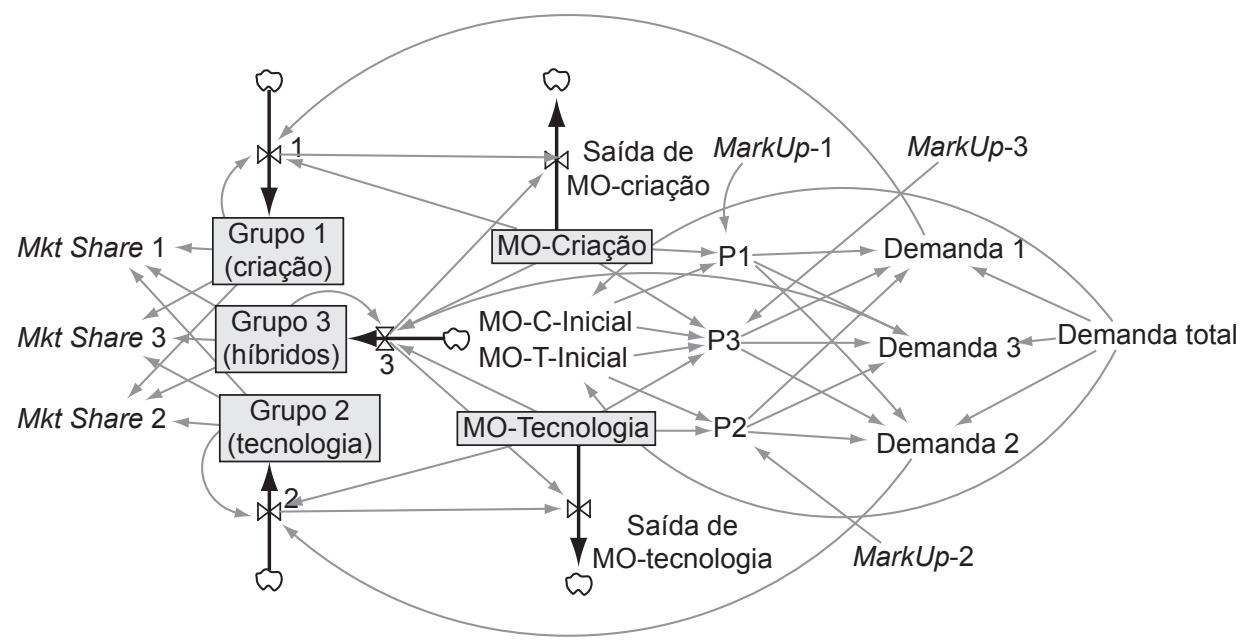

Figura 2. Modelo de simulação. Fonte: Ventana Systems, 2002. 
Se o Grupo 1 é incrementado em uma unidade, ou seja, adquire um novo cliente, o estoque de mão-deobra, MO-criação, perde uma unidade de mão-de-obra. Analogamente, se o Grupo 2 adquire um novo cliente, o estoque de mão-de-obra, MO-tecnologia, perde uma unidade de mão-de-obra. Contudo, se o Grupo 3 adquire um novo cliente, tanto o estoque de mão-deobra, MO-criação, quanto o estoque de mão-de-obra, MO-tecnologia, perdem uma unidade de mão-deobra.

Assim, esta modelagem reflete o fato de a disponibilidade de mão-de-obra definir a capacidade máxima de produção de cada grupo estratégico, pois no momento em que o estoque de mão-de-obra se esgotar, não será possível ampliar a oferta, ainda que haja demanda não atendida.

\subsubsection{Preço}

Os preços são afetados por duas variáveis fundamentais, quais sejam, a margem de lucro desejada (mark-up) e a disponibilidade de mão-de-obra para a prestação dos serviços (MO-criação e MO-tecnologia).

As margens de lucro de cada grupo estratégico são distintas e estão relacionadas com fatores alheios ao escopo deste trabalho, razão pela qual são tratadas como variáveis exógenas. Em decorrência do que já foi explanado anteriormente, constata-se que, em ordem crescente de margem de lucro, temos em primeiro lugar o Grupo 2, seguido pelo Grupo 1, e finalmente o Grupo 3 com a maior margem. Assim, foram definidos os seguintes valores para as margens de lucro de cada grupo:

$$
\begin{aligned}
& \text { MarkUp-2 }=1,2 \\
& \text { MarkUp-1 }=1,3 \\
& \text { MarkUp-3 }=1,4
\end{aligned}
$$

A mão-de-obra deste tipo de serviço é limitada e por este motivo uma variação na sua disponibilidade afeta diretamente os preços praticados, dado o aumento de custos para contratação e retenção de profissionais em momentos em que a disponibilidade de mão-de-obra diminui, conforme já exposto anteriormente. Quanto maior for a diferença entre a disponibilidade de mão-deobra em um dado momento e a disponibilidade existente no momento inicial, maior será o preço praticado, como demonstram as funções abaixo.

$$
\begin{aligned}
& \mathrm{P} 1=(((\text { (MO-C-Inicial"-"MO-Criação")/ } \\
& \text { "MO-C-Inicial”) + 0.5) * “MarkUp-1" } \\
& \text { P2 = (((“MO-T-Inicial"-"MO-Tecnologia")/ } \\
& \text { "MO-T-Inicial") + 0.5) * “MarkUp-2” }
\end{aligned}
$$

$$
\begin{aligned}
& \mathrm{P} 3 \text { = (((("MO-C-Inicial”+"MO-T-Inicial") - } \\
& \text { ("MO-Criação"+"MO-Tecnologia”))/ } \\
& \text { ("MO-C-Inicial”+"MO-T-Inicial")) + } \\
& \text { 0.5) * “MarkUp-3" }
\end{aligned}
$$

Para que o preço seja afetado pela variação do estoque de mão-de-obra disponível de forma adimensional, utiliza-se a transformação matemática acima de forma a garantir que o valor final do preço de cada grupo esteja dentro do intervalo $[0,5 ; 1,5]$ multiplicado pelo mark-up de cada grupo. Desta forma temos:

$$
\begin{aligned}
& 0,65 \leq \mathrm{P} 1 \leq 1,95 \\
& 0,60 \leq \mathrm{P} 2 \leq 1,80 \\
& 0,70 \leq \mathrm{P} 3 \leq 2,10
\end{aligned}
$$

\subsubsection{Demanda}

A demanda total do mercado é uma variável exógena. Uma vez que a análise se concentra nas participações de mercado de cada grupo, o valor absoluto da demanda é irrelevante para a obtenção dos resultados, razão pela qual esta variável foi arbitrariamente definida como 100.

A demanda de cada grupo estratégico foi estimada a partir de um modelo de Bertrand diferenciado, conforme apresentado por Shy (1995, p. 139). Neste caso, temos o que se segue:

$$
\begin{gathered}
\text { Demanda }= \\
\left(\mathrm{a}_{1}-\mathrm{b}_{1} * \mathrm{P}_{1}+\mathrm{c}_{1} * \mathrm{P}_{2}+\mathrm{d}_{1} * \mathrm{P}_{3}\right) * \text { Demanda Total, }
\end{gathered}
$$

sendo análogas as demandas dos grupos 2 e 3

Os coeficientes $\left(a_{i}, b_{i}, c_{i}\right.$ e $\left.d_{i}\right)$ foram obtidos a partir da observação direta do mercado, conforme apresentado na Tabela 1. Os coeficientes ai são iguais a 0,333 , pois assume-se que caso todos os preços fossem zero, as demandas de cada grupo seriam equivalentes a 1/3 da demanda total. Os coeficientes $b_{i}(0,25)$ são maiores do que $c_{i}(0,125)$ e $d_{i}$ $(0,125)$, pois os preços dos serviços de um determinado grupo estratégico são mais importantes para determinar sua demanda do que os preços dos grupos estratégicos concorrentes. Algumas das simulações exploram os efeitos das variações nesses coeficientes, que em termos econômicos correspondem a uma variação do grau de

Tabela 1. Coeficientes das funções de demanda.

\begin{tabular}{ccc}
\hline \multicolumn{3}{c}{ Demanda } \\
\hline $\mathbf{1}$ & $\mathbf{2}$ & $\mathbf{3}$ \\
\hline $\mathrm{a}_{1}=0,333$ & $\mathrm{a}_{2}=0,333$ & $\mathrm{a}_{3}=0,333$ \\
$\mathrm{~b}_{1}=0,250$ & $\mathrm{~b}_{2}=0,250$ & $\mathrm{~b}_{3}=0,250$ \\
$\mathrm{c}_{1}=0,000$ & $\mathrm{c}_{2}=0,000$ & $\mathrm{c}_{3}=0,125$ \\
$\mathrm{~d}_{1}=0,125$ & $\mathrm{~d}_{2}=0,125$ & $\mathrm{~d}_{3}=0,125$ \\
\hline
\end{tabular}


diferenciação dos serviços. Observa-se ainda, que os coeficientes $c_{1}$ e $c_{2}$ são iguais a zero porque os grupos $1 \mathrm{e}$ 2 não competem entre si.

Nota-se que os preços variam em função da disponibilidade de mão-de-obra e, conseqüentemente, as demandas também são afetadas por essa variação.

\subsubsection{Oferta}

Os estoques Grupo 1, Grupo 2 e Grupo 3 representam a oferta de cada grupo estratégico e são afetados pelos fluxos de novos clientes. Os fluxos são positivos enquanto as demandas forem maiores que a oferta e enquanto houver disponibilidade de mão-de-obra. Caso contrário, os fluxos serão negativos.

No momento $\mathrm{t}=0$, nenhum grupo possui clientes, e a disponibilidade de mão-de-obra é máxima. Como descrito anteriormente, ao longo do tempo cada grupo adquire novos clientes, ampliando sua oferta, até que seja atingido o equilíbrio de mercado (oferta $=$ demanda) ou até que se esgote a disponibilidade de mão-de-obra. Neste segundo caso, o grupo não conseguirá ampliar sua oferta, pois sua capacidade máxima de produção foi alcançada, ainda que exista demanda não atendida. Por fim, assume-se que um novo cliente gera um novo projeto e que este novo projeto demanda uma unidade de mão-de-obra para ser implantado, ou seja, não existem economias de escala.

\subsubsection{Análise gráfica do caso canônico}

Para facilitar a análise do comportamento de mercado a partir de um choque qualquer, apresenta-se o gráfico da situação inicial do modelo (Figura 3). O eixo das abscissas representa o tempo em semanas, enquanto o eixo das ordenadas representa as participações de mercado individualmente consideradas.

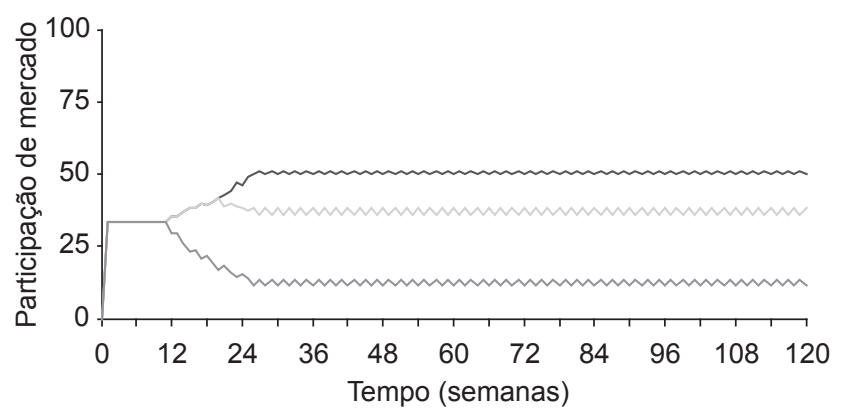

Market share - Grupo 1 (criação)

Market share - Grupo 2 (tecnologia)

Market share - Grupo 3 (híbridos)

Figura 3. Participação de mercado do caso canônico. Fonte: Ventana Systems, 2002.
Pode-se observar pelos gráficos que até o instante $\mathrm{t}=11$ as participações de mercado de cada grupo são iguais a 33,33\%. Nesse instante, o grupo 1 atingiu seu equilíbrio (demanda $1=$ oferta $1=11$ ). A partir daí, a sua participação de mercado diminuiu até se estabilizar em $11,54 \%$ (6 clientes).

Enquanto isso, os outros dois grupos continuaram a ampliar suas carteiras de clientes até o instante $\mathrm{t}=20$, momento em que o grupo 2 também atingiu o equilíbrio (demanda $2=$ oferta $2=20$ ). A partir deste momento a participação de mercado do grupo 2 diminuiu até se estabilizar em $38,46 \%$ ( 20 clientes).

A partir do instante $t=20$, apenas o grupo 3 ampliou a sua oferta, até que no instante $\mathrm{t}=26$ alcançou seu equilíbrio com participação de mercado de 50\% (26 clientes). É interessante notar que esse equilíbrio foi atingido em razão da indisponibilidade de mão-de-obra de criação, a despeito da demanda por projetos híbridos (29) ainda ser maior do que a oferta (26).

Por fim é importante dizer que, no equilíbrio, tanto o número de clientes quanto a participação de mercado varia (sobem e descem em uma unidade por período), pois as demandas variam a cada instante do tempo. Quando a demanda é maior do que a oferta, o grupo perde um cliente e quando a demanda é menor do que a oferta o grupo ganha um cliente. No equilíbrio, cada grupo ganha num instante e perde no instante seguinte, porém esta variação descreve exatamente o equilíbrio de mercado.

\subsubsection{Choques no modelo}

Esta seção apresenta uma síntese dos resultados das simulações em que há variação das variáveis exógenas, tendo o duplo propósito de testar a sensibilidade do modelo aos parâmetros utilizados em seu desenho, bem como identificar as variáveis mais relevantes para o entendimento da dinâmica concorrencial no setor de agências de internet. Foram feitas diversas simulações, como variação da demanda, nível de mark-up de cada grupo estratégico, grau de diferenciação entre os três grupos e acesso a recursos humanos qualificados. A seguir, dada a impossibilidade de apresentação de todos os resultados, é reportada a síntese daqueles mais contra-intuitivos. Demais simulações, assim como as rotinas utilizadas para a simulação, podem ser obtidas diretamente com os autores.

\subsubsection{Mudança no grau de diferenciação entre o grupo 1 e o grupo 3}

Modificações no grau de diferenciação dos grupos estratégicos podem ser implementadas por meio de investimentos em tecnologia ou propaganda, com conseqüente mudança de percepção dos clientes. A fim de simular quaisquer dessas mudanças, pode-se alterar o valor dos coeficientes $c_{i}$ e $d_{i}$. 
Na Figura 4, foi simulado um choque no instante $\mathrm{t}=60$, para refletir uma mudança de percepção quanto ao grau de diferenciação entre os grupos 1 (criação) e 3 (híbridos). Para isso, foi realizada uma redução de $50 \%$ no coeficiente $d_{1}$ (grau de diferenciação entre G1 e G3) e no coeficiente $c_{3}$ (grau de diferenciação entre G3 e G1), que passaram de 0,125 para 0,0625 .

Observa-se que no instante $\mathrm{t}=60$ (momento do choque), o aumento de $50 \%$ no grau de diferenciação $\mathrm{d}_{1}$ e $\mathrm{c}_{3}$ ocasionou uma redução no volume de clientes atendidos pelo grupo 1 do grupo 3. Essas reduções refletiram em um aumento na participação de mercado do grupo 2, fazendo com que este grupo passasse a ser líder de mercado, com uma participação de mercado maior do que a do grupo 3 .

O aumento no grau de diferenciação entre os grupos 1 e 3 reduziu significativamente as demandas de ambos. Essa diminuição é conseqüência direta da redução do componente $\left(\mathrm{c}_{\mathrm{i}}{ }^{*} \mathrm{P}_{\mathrm{i}}\right)$. Conclui-se que, nesse caso, o aumento no grau de diferenciação, contrariando as expectativas, atuou negativamente sobre os grupos analisados.

De maneira análoga, foi realizada simulação com a finalidade de refletir mudanças na percepção do grau de diferenciação entre os grupos 2 (tecnologia) e 3 (híbridos), através de uma redução de $50 \%$ nos coeficientes $d_{2}$ (grau de diferenciação entre G2 e G3) e $d_{3}$ (grau de diferenciação entre G3 e G2), que passaram de 0,125 para 0,0625 , no instante $t=60$ (Figura 5).

Constata-se que, a partir do instante $\mathrm{t}=60, \mathrm{o}$ aumento de $50 \%$ no grau de diferenciação $\mathrm{d}_{2}$ e $\mathrm{d}_{3}$ produziu reduções no volume de clientes para o grupo 2 e para o grupo 3. Essas reduções modificaram as participações de mercado, elevando a fatia do grupo 1 , diminuindo a participação do grupo 2 e incrementando a do grupo 3 .
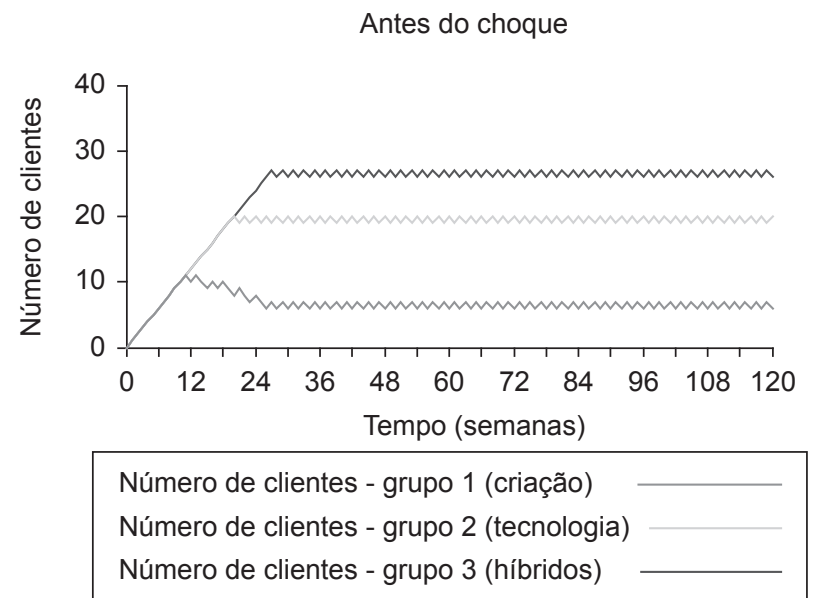

$\mathrm{G} 1=6, \mathrm{G} 2=20, \mathrm{G} 3=26$

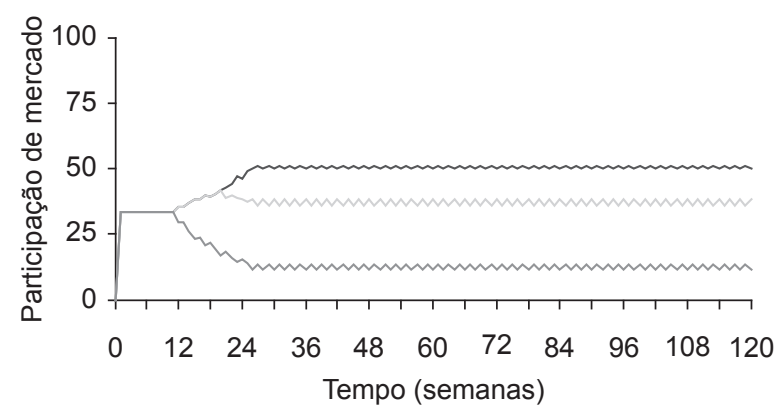

Market share - grupo 1 (criação)
Market share - grupo 2 (tecnologia)
Market share - grupo 3 (híbridos)

$\mathrm{G} 1=11,54 \%, \mathrm{G} 2=38,46 \%, \mathrm{G} 3=50,00 \%$
Depois do choque

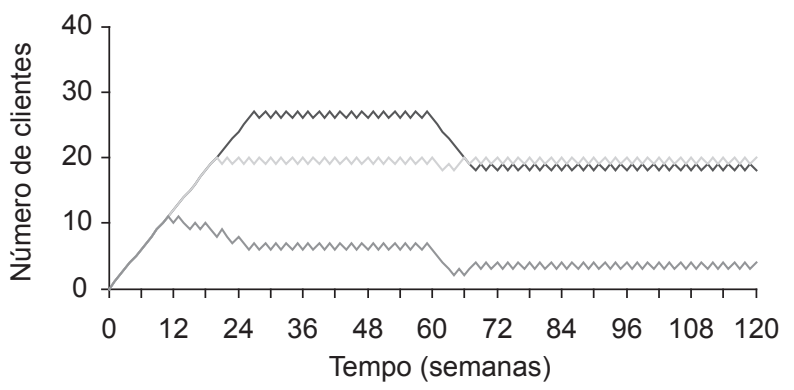

Número de clientes - grupo 1 (criação)

Número de clientes - grupo 2 (tecnologia)

Número de clientes - grupo 3 (híbridos)

$\mathrm{G} 1=4, \mathrm{G} 2=20, \mathrm{G} 3=18$

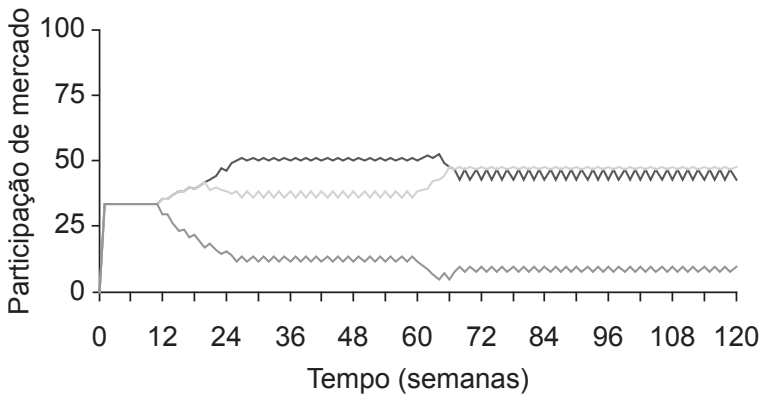

Market share - grupo 1 (criação)

Market share - grupo 2 (tecnologia)

Market share - grupo 3 (híbridos)

$\mathrm{G} 1=9,52 \%, \mathrm{G} 2=47,62 \%, \mathrm{G} 3=42,86 \%$

Figura 4. Mudança no grau de diferenciação entre o grupo 1 e o grupo 3. Fonte: Ventana Systems, 2002. 
Antes do choque

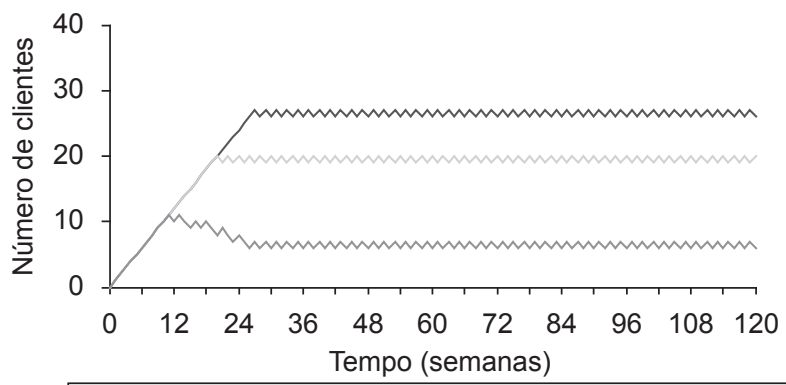

Número de clientes - grupo 1 (criação)

Número de clientes - grupo 2 (tecnologia)

Número de clientes - grupo 3 (híbridos)

$\mathrm{G} 1=6, \mathrm{G} 2=20, \mathrm{G} 3=26$

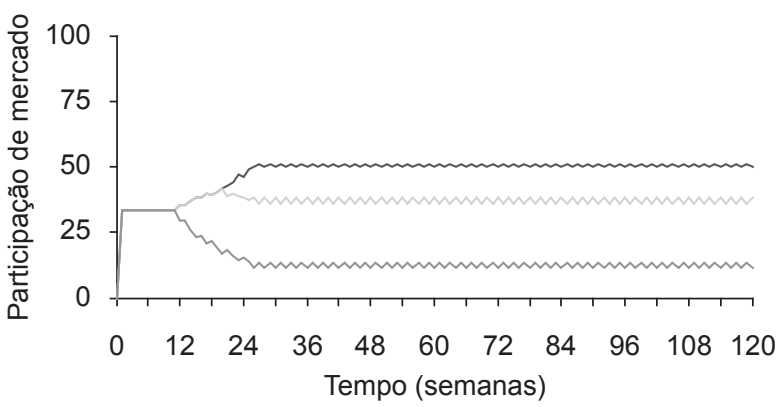

Market share - Grupo 1 (criação)

Market share - Grupo 2 (tecnologia)

Market share - Grupo 3 (híbridos)

$\mathrm{G} 1=11,54 \%, \mathrm{G} 2=38,46 \%, \mathrm{G} 3=50,00 \%$
Depois do choque

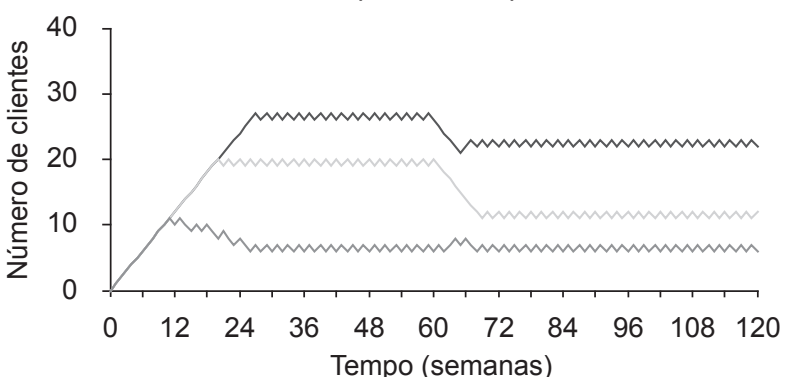

Número de clientes - grupo 1 (criação)

Número de clientes - grupo 2 (tecnologia)

Número de clientes - grupo 3 (híbridos)

$\mathrm{G} 1=6, \mathrm{G} 2=12, \mathrm{G} 3=22$

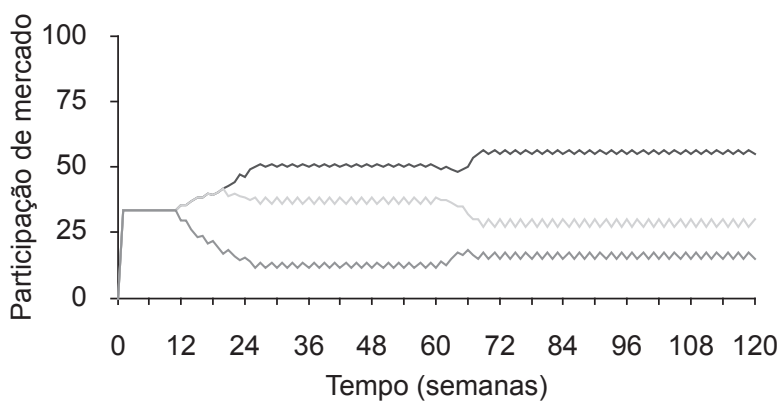

Market share - Grupo 1 (criação)

Market share - Grupo 2 (tecnologia)

Market share - Grupo 3 (híbridos)

$\mathrm{G} 1=15,00 \%, \mathrm{G} 2=30,00 \%, \mathrm{G} 3=55,00 \%$

Figura 5. Mudança no grau de diferenciação entre o grupo 2 e o grupo 3. Fonte: Ventana Systems, 2002.

É interessante notar, que apesar de os coeficientes de diferenciação serem idênticos para todos os grupos, as duas simulações geraram resultados muito distintos. Verifica-se, pela primeira simulação, que o aumento no grau de diferenciação dos grupos 1 e 3 reduziu a participação do grupo 3. Porém, esse mesmo aumento no grau de diferenciação, quando relativo aos grupos 2 e 3, surtiu efeito contrário, elevando a participação de mercado do grupo 3.

Esses resultados tão díspares são decorrência dos estoques de mão-de-obra existentes em cada um dos casos, no momento do choque. Enquanto no primeiro caso não havia mão-de-obra criativa disponível no mercado, no segundo caso a mão-de-obra tecnológica existia. Essa circunstância gerou alterações distintas nas demandas, em cada simulação, provocando tais resultados aparentemente contraditórios.

\subsubsection{Aumento da oferta de recursos humanos de criação}

Nesse tópico, foi aplicado um choque, quando o mercado de mão-de-obra de criação recebeu fluxo de vinte unidades de novos profissionais, no instante $\mathrm{t}=60$ (Figura 6).

Neste caso, no instante $t=60$, o estoque de mão-deobra de criação recebeu 20 unidades de profissionais, gerando assim uma redução nos preços dos grupos 1 e 3 , afetados por esta variação, e ocasionando mudanças nas demandas dos três grupos. O novo equilíbrio se deu com um aumento de quase 12 pontos percentuais na participação de mercado do grupo 1, e conseqüentes reduções de mais de 9 pontos percentuais e quase 4 pontos percentuais dos grupos 2 e 3 , respectivamente.

É interessante notar que, ao contrário do que se esperava, o aumento na oferta de mão-de-obra de criação gerou uma redução da participação de mercado do grupo 3 . Isso se deve ao aumento de $133 \%$ na oferta experimentada pelo grupo 1 (de 6 para 14 clientes), muito superior 
Antes do choque

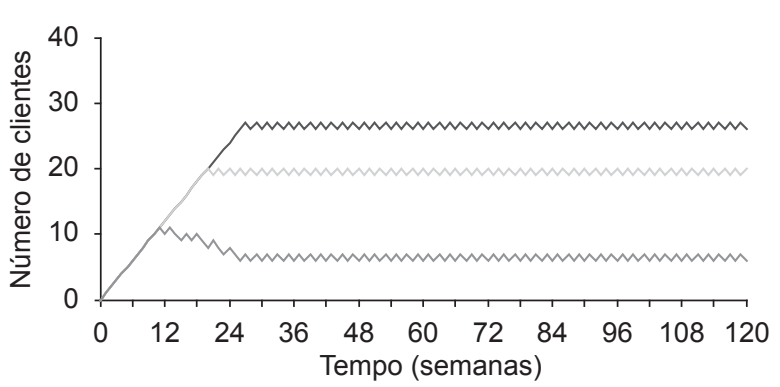

Número de clientes - grupo 1 (criação)

Número de clientes - grupo 2 (tecnologia)

Número de clientes - grupo 3 (híbridos)

$\mathrm{G} 1=6, \mathrm{G} 2=20, \mathrm{G} 3=26$

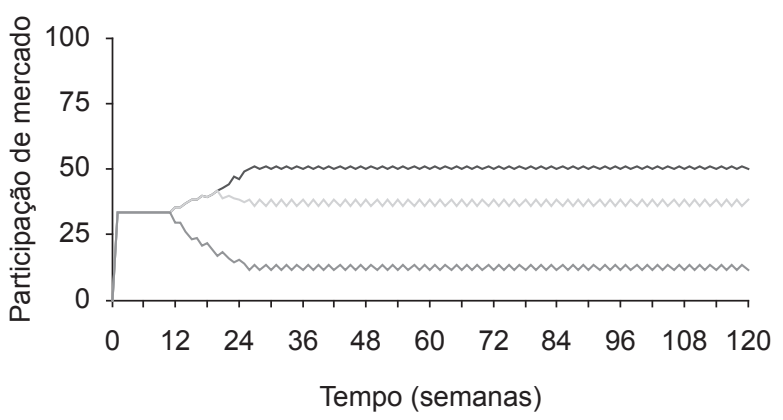

Market share - Grupo 1 (criação)

Market share - Grupo 2 (tecnologia)

Market share - Grupo 3 (híbridos)

$\mathrm{G} 1=11,54 \%, \mathrm{G} 2=38,46 \%, \mathrm{G} 3=50,00 \%$
Depois do choque

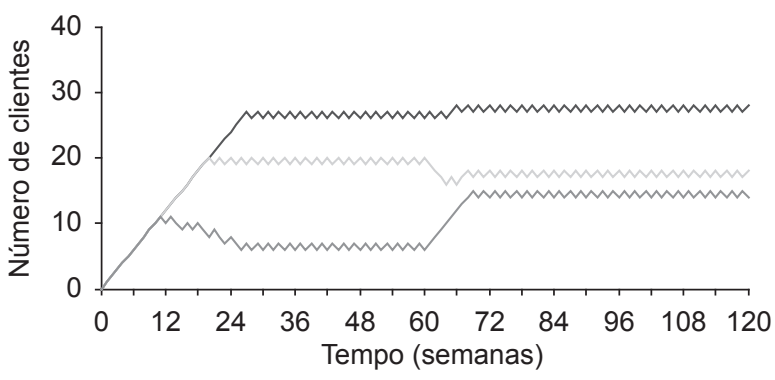

Número de clientes - grupo 1 (criação)

Número de clientes - grupo 2 (tecnologia)

Número de clientes - grupo 3 (híbridos)

$\mathrm{G} 1=14, \mathrm{G} 2=18, \mathrm{G} 3=28$

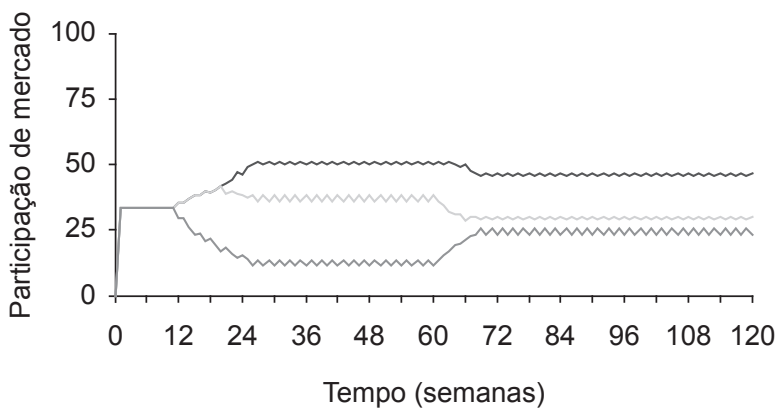

Market share - Grupo 1 (criação)

Market share - Grupo 2 (tecnologia)

Market share - Grupo 3 (híbridos)

$\mathrm{G} 1=23,33 \%, \mathrm{G} 2=30,00 \%, \mathrm{G} 3=46,67 \%$

Figura 6. Aumento da oferta de mão-de-obra de criação. Fonte: Ventana Systems, 2002.

ao aumento da oferta do grupo 3, de apenas $8 \%$ (de 26 para 28 clientes).

O aumento da oferta de mão-de-obra de criação alterou a demanda do grupo 3 com menos intensidade se comparado com a demanda do grupo 1 , pois a primeira também é afetada por variações do estoque de mão-de-obra de tecnologia, não modificado neste choque.

Nota-se também que, diferentemente deste caso, quando ocorre aumento da oferta de recursos humanos na área tecnológica (simulação não reportada), os dois grupos estratégicos que fazem uso deste recurso tiveram acréscimos em suas participações de mercado. Os diferentes resultados observados são decorrência da existência, ou não, de disponibilidade de mão-de-obra no momento do choque. A contradição foi gerada, pois no primeiro caso não havia mão-de-obra criativa disponível no mercado, enquanto que no segundo havia disponibilidade de mão-de-obra tecnológica.

\subsection{Introdução de variáveis estocásticas}

A introdução de variáveis estocásticas no modelo não alterou as principais conclusões a respeito do equilíbrio competitivo. Entretanto, como era de se esperar, houve aumento da volatilidade, e sobretudo do tempo necessário para que o modelo atingisse o equilíbrio. Esse resultado é mais plausível que o obtido pelo modelo determinístico, visto que choques inesperados retiram um sistema do equilíbrio, de tal modo que este é um estado via de regra não observado.

Ocomportamento estocástico foi introduzido nos fluxos de projetos a partir da utilização de uma função matemática chamada "INTEGER (RANDOM NORMAL)", que gera um número inteiro aleatório, entre o valor mínimo $\{$ min\} e o valor máximo $\{$ max $\}$, com média \{mean\} e desvio padrão $\{$ stdev $\}$. Se a oferta de um determinado grupo estratégico for maior do que sua demanda, ou se não houver disponibilidade de mão-de-obra, então: $\{\min \}$ 
$=-3,\{\max \}=1,\{$ mean $\}=-1$ e $\{$ stdev $\}=1$, ou seja, há uma pequena probabilidade de que este grupo aumente sua oferta. Por outro lado, se a oferta de um determinado grupo estratégico for menor do que sua demanda e existir disponibilidade de mão-de-obra, então: $\{\min \}=-1,\{\max \}$ $=3,\{$ mean $\}=1$ e $\{$ stdev $\}=1$, ou seja, existe uma grande probabilidade de aumento de oferta deste grupo.

As variáveis estocásticas inseridas no modelo impedem propositalmente que os grupos estratégicos sejam capazes de adquirir mais do que dois novos projetos num dado período, convencionado em uma semana, limite baseado em observações empíricas que demonstram que as firmas não conseguem trabalhar acima desse nível. Justifica-se tal circunstância em razão das equipes de vendas terem foco nos processos de negociação de novas contas (contratos, contratação de equipes e consultoria) e, portanto, não conseguirem se dedicar ao trabalho de novas prospecções.

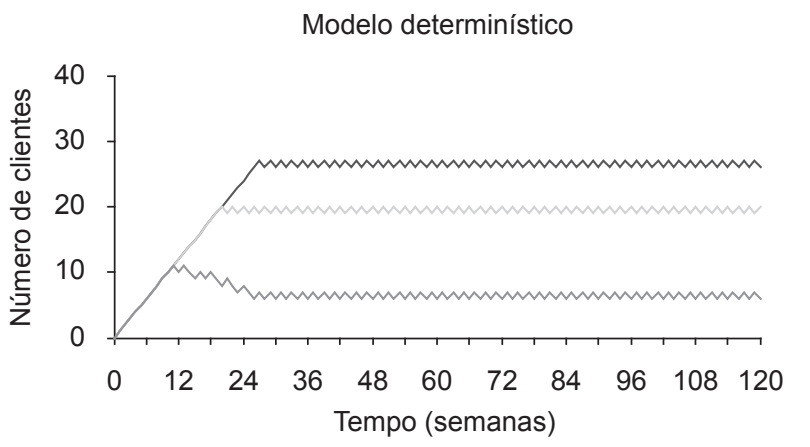

Número de clientes - grupo 1 (criação)
Número de clientes - grupo 2 (tecnologia)
Número de clientes - grupo 3 (híbridos)

$\mathrm{G} 1=6, \mathrm{G} 2=20, \mathrm{G} 3=26$

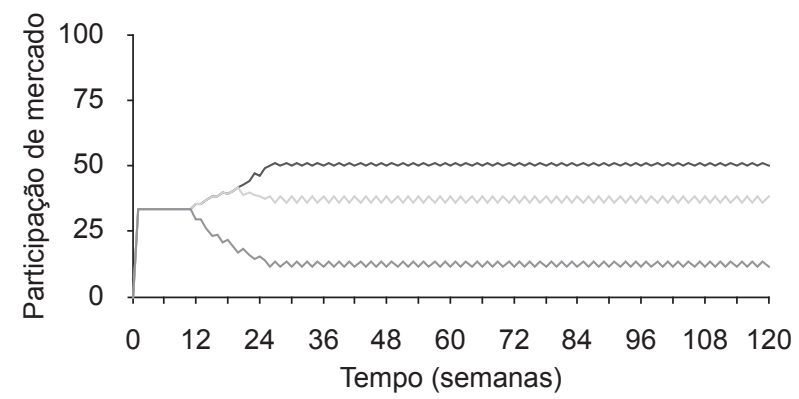

Market share - Grupo 1 (criação)

Market share - Grupo 2 (tecnologia)

Market share - Grupo 3 (híbridos)

$\mathrm{G} 1=11,54 \%, \mathrm{G} 2=38,46 \%, \mathrm{G} 3=50,00 \%$
Pode-se observar na Figura 7, que o resultado observado no modelo estocástico é semelhante ao do determinístico, porém, a inclusão das variáveis estocásticas gerou um comportamento mais volátil no segundo modelo. Além disso, constata-se que no modelo estocástico os grupos levaram mais tempo para atingir seus equilíbrios.

\section{Conclusões}

O setor de agências de internet é uma indústria bastante particular, em que as barreiras de capital mostram-se pouco relevantes à dinâmica concorrencial. Este trabalho evidenciou, por meio de simulação de dinâmica de sistemas, que o recurso mais relevante para a concorrência entre os três grupos estratégicos - de criação, de base tecnológica e híbridos - é o acesso a recursos humanos qualificados, os quais são irreproduzíveis no curto prazo e definem a capacidade de produção de cada grupo estratégico.

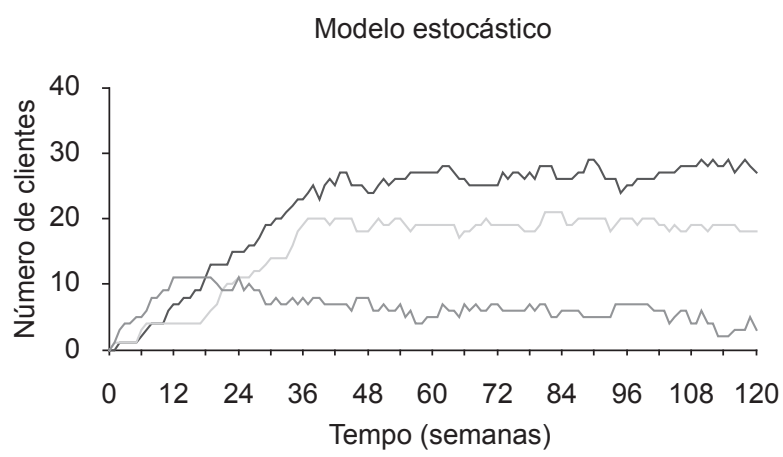

Número de clientes - grupo 1 (criação)

Número de clientes - grupo 2 (tecnologia)

Número de clientes - grupo 3 (híbridos)

$\mathrm{G} 1=3, \mathrm{G} 2=18, \mathrm{G} 3=27$

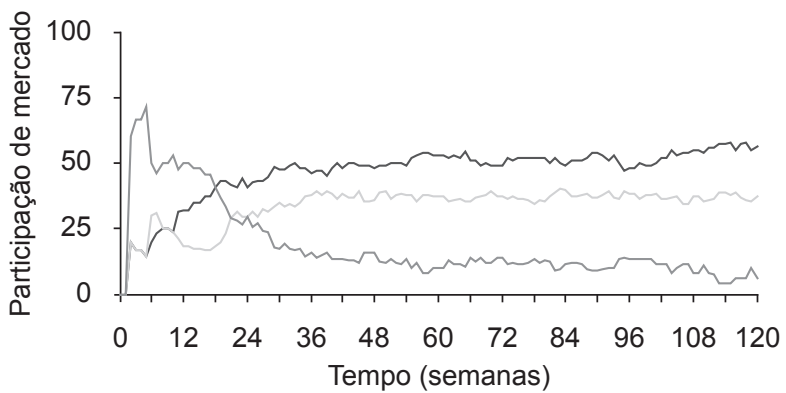

Market share - Grupo 1 (criação) Market share - Grupo 2 (tecnologia) Market share - Grupo 3 (híbridos)

$\mathrm{G} 1=6,25 \%, \mathrm{G} 2=37,50 \%, \mathrm{G} 3=56,25 \%$

Figura 7. Comparação gráfica dos modelos determinístico e estocástico. Fonte: Ventana Systems, 2002. 
De um modo geral, as simulações apresentaram resultados intuitivos, particularmente no que se refere aos efeitos de variabilidade da demanda e níveis do mark-up de cada grupo estratégico. A simulação, entretanto, adiciona a esses resultados intuitivos a idéia de magnitude e timing de resposta da participação de mercado às diversas variáveis que foram objeto de simulação.

Entre os resultados contra-intuitivos, destaca-se o efeito das mudanças no grau de diferenciação entre os diferentes grupos. O aumento no grau de diferenciação entre os grupos de criação e híbridos produziu resultados distintos daqueles obtidos quando foi aumentado o grau de diferenciação entre os grupos de tecnologia e híbridos. No primeiro caso, houve redução na participação de mercado do grupo híbrido, tendo sido observado resultado inverso no segundo caso. Essa diferença decorre da maior elasticidade da oferta de recursos humanos voltados ao grupo de tecnologia, a partir da disponibilidade em outras indústrias (softwares, tecnologia de informação, análise de sistemas, entre outras), que compartilham o mesmo recurso humano. $\mathrm{O}$ aumento da diferenciação do grupo de criação faz com que este absorva os recursos humanos de criação, reduzindo a capacidade de concorrência do grupo híbrido, que tem que disputar esse recurso escasso. No caso da diferenciação entre os segmentos de tecnologia e híbridos, a maior disponibilidade de recursos humanos de base tecnológica, em indústrias correlatas, permite o crescimento da participação dos dois grupos.
É por essa mesma particularidade anteriormente descrita, qual seja, indisponibilidade de recursos humanos de criação, que um choque da sua oferta afeta as participações de mercado de forma diferente da verificada quando há choque de oferta de recursos humanos de base tecnológica. O aumento de recursos humanos de criação gerou uma redução na participação de mercado do grupo de empresas híbridas, enquanto o aumento de recursos humanos de tecnologia produziu um resultado inverso.

Entre as limitações do modelo de simulação, destaca-se o pressuposto de interação entre grupos estratégicos, representados como unidades decisórias. Essa representação deliberadamente não trata da interação estratégica dentro de cada grupo, o que permitiria tornar variáveis endógenas como as respostas às mudanças de preços e estratégias de diferenciação. Essa limitação, entretanto, não retira o mérito da análise da interação entre grupos estratégicos, sobretudo no caso do setor de agências de internet, que por sua insipiência, ainda não foi objeto de análises de concorrência. Mesmo assim, é recomendável a ampliação do modelo em pesquisas futuras, por meio da inserção de mais de uma firma em cada grupo estratégico e da definição de um modelo de concorrência entre estas firmas. Desse modo, seria possível avaliar o efeito de variáveis endógenas sobre o padrão de comportamento do grupo.

\title{
internet agencies industry in Brazil: competition analysis using system dynamics
}

\begin{abstract}
This paper aims to analyze the dynamics of market share of different strategic groups that are part of the internet agencies industry in Brazil as well as the changes due to modifications in its critical variables such as price, advertising and technology investment, and the availability of qualified human resources. For that reason the method of computer simulation, using system dynamics, was adopted. The adoption of this technique allowed identifying non-trivial patterns, especially the ones related to the analyses of the changes in the degree of differentiation between the different strategic groups.
\end{abstract}

Keywords: Internet. Software industry. System dynamics. Differentiated bertrand. 
CARPANEZ, J. Brasil tem 24 milhões de computadores em uso, diz FGV. Folha de São Paulo, São Paulo, 30 mar. 2005. Disponível em: <http://www1.folha.uol.com.br/folha/ informatica/ ult124u18231.shtml>. Acesso em: 23 out. 2005.

CGI. Comitê Gestor de internet no Brasil. Histórico sobre a internet no Brasil. Brasília, 2005. Disponível em: <http://www. cg.org.br/sobre-cg/historia.htm>. Acesso em: 12 nov. 2005.

FORRESTER, J. W. Industrial dynamics. Massachusetts: Pegasus Communications, 1999. 464 p.

FORRESTER, J. W. The beginning of system dynamics. Massachusetts, 1996. Disponível em: <http://web.mit.edu/sdg/ www/Papers/D-4165-1.pdf>. Acesso em: 12 fev. 2005.

LAUDON, K. C.; LAUDON, J. P. Sistemas de informação. 4. ed. Rio de Janeiro: LTC Livros Técnicos e Científicos, 1999. 389 p. Tradução: Dalton Conde de Alencar. Revisão técnica: Cristina Bacelar

LOVELOCK, C. H. Services marketing. 3 ed. New Jersey: Prentice-Hall, 1996. 660 p.

MILGRON, P. R.; ROBERTS, J. Economics, organization and management. New Jersey: Prentice-Hall, 1992. 621 p.
PORTER, M. E. Estratégia competitiva: técnicas para análise de indústrias e da concorrência. 7. ed. Rio de Janeiro: Campus, 1986. 362 p. Tradução: Elizabeth Maria de Pinho Braga. Revisão técnica: Jorge A. Garcia Gómez.

SHAPIRO, C.; VARIAN, H. R. Economia da informação: como os princípios econômicos se aplicam à era da internet. 7. ed. Rio de Janeiro: Elsevier, 1999. 397 p. Tradução: Ricardo Inojosa.

SHY, O. Industrial Organization: theory and applications. Massachusetts: MIT Press, 1995. 466 p.

VARIAN, H. R. Microeconomia: princípios básicos. 6. ed. Rio de Janeiro: Campus, 2003. 778 p. Tradução: Maria José Cyhlar Monteiro. Revisão técnica: Lia Hasenclever.

VENTANA SYSTEMS, INC. Causal tracing: causal tracing enables fast and accurate analysis of model dynamics. Massachusetts, 2002. Disponível em: <http://www.vensim. com/causal.html>. Acesso em: 04 nov. 2004.

VENTANA SYSTEMS, INC. Vensim user's guide: version 5. Massachusetts, 2002. Disponível em: <http://www.vensim. com/ffiles/VensimUsersGuide.zip>. Acesso em: 03 nov. 2004.

\section{Sobre os autores}

\section{Antony José Souza Martins}

Escola de Economia de São Paulo - EESP, Fundação Getulio Vargas - FGV, Rua Aimberé, 909, apto 124, Perdizes, CEP 05018-011, São Paulo, SP, Brasil, e-mail: antony@tv1.com.br

\section{Paulo Furquim de Azevedo}

Escola de Economia de São Paulo - EESP, Fundação Getulio Vargas - FGV, Rua Itapeva, 474, sl 1207, Bela Vista, CEP 01332-000, São Paulo, SP, Brasil, e-mail: paulo.azevedo@fgv.br 